Review

\title{
Robotics in Endometrial Cancer Care
}

\section{Joseph Ng}

Department of Obstetrics and Gynecology, National University Health System, Singapore 119228, Singapore; E-Mail: joseph_ng@nuhs.edu.sg; Tel.: +65-6779-5555; Fax: +65-6779-4373

Received: 25 September 2013; in revised form: 4 November 2013 / Accepted: 6 November 2013 / Published: 12 November 2013

\begin{abstract}
Endometrial cancer is the most common gynecological cancer in women in most of the developed world. The majority of these women with endometrial cancer will be unaffected by their disease. The challenge therefore is for surgical treatment not to be worse than the disease. Robotics has changed the way that we care for women living with endometrial cancer by making low-impact surgical treatment available to more women than was previously possible.
\end{abstract}

Keywords: robotics; endometrial; cancer; laparoscopy

\section{Introduction}

Endometrial cancer is the most common gynecological cancer in women in most developed countries [1-4]. There is a strong epidemiological association between demographic characteristics of obesity, lower parity (the number of times a woman has been pregnant), earlier menarche (age of the onset of menses) and late menopause (age at which menses ceases) and the risk of endometrial cancer. These demographic risk factors characterize a significant proportion of women in developed countries $[2,4]$. Although endometrial cancer is the most common gynecological cancer, it is not the most deadly. Women who have a diagnosis of endometrial cancer can expect to be completely cured with a very low lifetime risk of recurrent endometrial cancer. This is because $85 \%$ of endometrial cancer is diagnosed in the first stage of disease [3]. This means that at the time of diagnosis, the cancer is confined entirely to the endometrium with no evidence of extra-uterine disease. Early detection is possible because early changes in the endometrium result in abnormal vaginal bleeding, which prompts women to seek medical attention. Therefore, it is common at the time of the diagnosis of endometrial cancer to find that other than abnormal vaginal bleeding, the patient is unaffected by the 
disease. The woman living with endometrial cancer is often an active and productive member of society, contributing to the economy, an integral part of a nuclear family and an otherwise healthy individual.

The standard of care in endometrial cancer is surgery to remove the uterus, Fallopian tubes and ovaries, a procedure known as a total hysterectomy with bilateral salpingo-oophorectomy. The regional lymph nodes may also need to be removed surgically depending on the characteristics of the primary endometrial cancer. In early stage endometrial cancer, total hysterectomy and bilateral salpingo-oopherectomy is sufficient treatment. Therefore, the challenge in the clinical management of most cases of endometrial cancer is to minimize the impact of surgical treatment, which often is more debilitating than early endometrial cancer that has little or no functional effect on the patient.

In contemporary practice, options available to patients for endometrial cancer surgical treatment are open surgery, traditional laparoscopic surgery or robot-assisted surgery. There is data to suggest that the standard of care for endometrial cancer is laparoscopic surgery $[5,6]$.

\section{The Surgical Treatment of Endometrial Cancer: A Brief History}

Open surgery remains the most intuitive and direct way in which a surgeon accesses the abdominal and peritoneal cavity. Open surgery affords the surgeon the space and access to operate directly in the abdominal cavity with hands and instruments. Open surgery is where most surgeons first achieve surgical proficiency and remains the default mode of surgery when technical difficulty makes other modes of surgery less attractive or impossible. The primary disadvantage of open surgery is the open wound that is created to facilitate surgery and from which the patient then has to recover. The more extensive the surgical wound, the higher the rate of complications that accompany surgical recovery. Complications, such as poor wound healing, infection and bleeding, become more common the larger the surgical wound. Particularly relevant to endometrial cancer patients is the high incidence of diabetes mellitus that further increases the risk of poor wound healing and infection above that of the general population. Recovery from open surgery is also associated with significant pain and discomfort [5]. This pain and discomfort prevents prompt resumption of preoperative levels of activity and function, from normal breathing to the conduct of the essential activities of daily life. This decreased function due to pain increases the patient's risk of thromboembolic and infectious complications postoperatively.

Hysterectomy with laparoscopic assistance was first formally described by Reich et al. in 1989 [7]. With the advent of laparoscopy, women who needed gynecological surgery had the option of having surgical treatment that was much less invasive, primarily without the need to recover from a significant surgical wound to the abdomen. This was particularly significant in the treatment of endometrial cancer, where surgical treatment for the cancer could be much less invasive, taking the standard of care that much closer to the ideal of effectively treating the cancer without affecting the function of an otherwise healthy individual. However, almost 30 years on, the bulk of hysterectomies are still performed through traditional open laparotomy incisions with the rates of laparoscopic hysterectomy between $4 \%$ and $30 \%$ [8-10]. The rates of laparoscopic hysterectomy may have reached a relative steady state, due primarily to two factors: the performance characteristics of laparoscopy as a surgical technique and patient factors. Laparoscopy is not an intuitive way to operate; the performance characteristics of the instruments means that they behave in a way that is often counter-intuitive, which surgeons compensate for with experience and training. Having to compensate for the fulcrum effect 
and the use of smaller, non-articulating instruments are just some examples of the barriers to intuitively performing surgery. Laparoscopy requires that the surgeon who has achieved proficiency at open surgery take one step backwards to re-learn surgical technique to compensate for the performance characteristics of laparoscopy. Quite simply, the surgeon has to compensate for the awkward mechanics of laparoscopic surgery, so that the patient can benefit from a less invasive procedure. This also means that as the surgical complexity increases, there is an accompanying exponential increase in the level of difficulty in performing the surgery laparoscopically. Consequently, surgeons often fall back on their proficiency in open surgery when laparoscopic cases become too difficult, as is the case in systematic lymphadenectomy or when the anatomy is distorted in surgery for cancer [10]. There is also a significant learning curve associated with acquiring laparoscopic proficiency [11]. The resultant effect of these factors is that expertise in laparoscopic cancer surgery is not as widespread as it should be, centered mainly in academic centers [2]. Ultimately, this limits patients' access to minimally invasive cancer surgery, and for women with endometrial cancer, this means not always having the option of having cancer treatment without significantly affecting their ability to function.

\section{Robotics in Endometrial Cancer}

The daVinci ${ }^{\circledR}$ surgical robotic platform grew out of defense programs in the 1980 s to develop technology that would support remote surgery, thereby projecting the surgeon's expertise forward into the frontline, where this expertise would be needed without exposing the surgeon to the hazards of the battle front. Today, the emphasis in robotic surgery is less about remote projection or "telepresence" and more about the ability of the robot to enhance the surgeon's existing skill sets. The robotic instruments are built to mimic the motion of the human hand, albeit in a very limited fashion, with rotation and limited supination and pronation type articulation at the "wrist" of the instrument. The instrumentation also filters out physical noise, such as the resting tremor of the human hand and provides consistent physical strength and power that at the scale of the instrument exceeds that of the full-sized human hand. All these simple innovations allow the surgeon to operate in a natural manner, moving instruments and robotic "hands" in a fashion that closely mirrors open surgical maneuvers. As such, there is minimal "re-learning" involved, and the move from open surgery to robotic surgery is more of a step rather than the jump required in laparoscopy. This gentler learning curve is what is attracting surgeons to robotic surgery on a scale not seen when laparoscopy was introduced into gynecological surgical practice. This surge in the uptake of robotic surgery translates into increased patient access to minimally invasive gynecological surgery $[12,13]$. Robotics has leveled the playing field by removing the "work" of overcoming the awkwardness of laparoscopy with technology. In essence, the use of machines has made work easy once again. Robotic surgery is such an attractive option to most gynecological surgeons, that recent reports have suggested that it has perhaps been "overused" since its introduction after FDA approval for gynecological surgery in 2005 [14]. One can therefore make two important inferences: first, that relatively small incremental increases in the physical performance of existing surgical instrumentation can result in an exponentially better operator experience; second, that these same small increments in performance can dramatically improve the access that women have to minimally invasive surgical care, who would have otherwise only have had the option of traditional open surgery. 


\section{Conclusion}

Robotic surgery is the latest iteration in gynecological surgical treatment that takes us one step closer to the Holy Grail of zero-impact surgical treatment. Robotic surgery's application in the surgical treatment of endometrial cancer is timely for the following reasons. First, endometrial cancer incidence is rising and is often the most common gynecological cancer in developed countries. Second, the majority of women who are diagnosed with endometrial cancer have early stage disease and, so, are functionally unaffected by their disease. Third, the majority of endometrial cancer surgery is still being performed via open laparotomy, which, as far as the patient is concerned, is a situation in which the "treatment is worse than the disease". Fourth, laparoscopy, although associated with good outcomes and much less invasive than open surgery, should be the standard of care, but due to its performance characteristics, is unpopular and, therefore, not as accessible as the standard of care should be. Finally, robotic surgery is both easy to adopt, powerful and is associated with good clinical outcomes when adopted by open surgeons [15] with the potential to lower the overall cost of endometrial cancer care [16,17], but it is plagued at present by the issue of cost. In my view, it is reasonable to assume that the largely technology-associated cost of robotic surgery will fall, and once the obstacle of cost is breached, most surgeons will have access to the advantages of robot-assisted surgery and be able to offer minimally invasive options to patients, where there were none, thereby improving the standard of care for one of the most common gynecological cancers in the world.

\section{Acknowledgments}

We thank Wong Peang Cheang and Yong Eu Leong, for their steadfast support in bringing robotic surgical care to all the women for whom we care.

\section{Conflicts of Interest}

The author declares no conflict of interest.

\section{References}

1. Lindemann, K.; Eskild, A.; Vatten, L.J.; Bray, F. Endometrial cancer incidence trends in Norway during 1953-2007 and predictions for 2008-2027. Int. J. Cancer 2010, 127, 2661-2668.

2. Evans, T.; Sany, O.; Pearmain, P.; Ganesan, R.; Blann, A.; Sundar, S. Differential trends in the rising incidence of endometrial cancer by type: Data from a UK population-based registry from 1994 to 2006. Br. J. Cancer 2011, 104, 1505-1510.

3. Duong, L.M.; Wilson, R.J.; Ajani, U.A.; Singh, S.D.; Eheman, C.R. Trends in endometrial cancer incidence rates in the United States, 1999-2006. J. Women Health 2011, 20, 1157-1163.

4. Bray, F.; Dos Santos Silva, I.; Moller, H.; Weiderpass, E. Endometrial cancer incidence trends in Europe: Underlying determinants and prospects for prevention. Cancer Epidemiol. Biomark. Prev. 2005, 14, 1132-1142. 
5. Janda, M.; Gebski, V.; Brand, A.; Hogg, R.; Jobling, T.W.; Land, R.; Manolitsas, T.; McCartney, A.; Nascimento, M.; Neesham, D. Quality of life after total laparoscopic hysterectomy versus total abdominal hysterectomy for stage I endometrial cancer (LACE): A randomised trial. Lancet Oncol. 2010, 11, 772-780.

6. Walker, J.L.; Piedmonte, M.R.; Spirtos, N.M.; Eisenkop, S.M.; Schlaerth, J.B.; Mannel, R.S. Barakat, R.; Pearl, M.L.; Sharma, S.K. Recurrence and survival after random assignment to laparoscopy versus laparotomy for comprehensive surgical staging of uterine cancer: Gynecologic Oncology Group LAP2 Study. J. Clin. Oncol. 2012, 30, 695-700.

7. Reich, H. New techniques in advanced laparoscopic surgery. Bailliere's Clin. Obstet. Gynaecol. 1989, 3, 655-681.

8. Patel, P.R.; Lee, J.; Rodriguez, A.M.; Borahay, M.A.; Snyder, R.R.; Hankins, G.D.; Kilic, G.S. Disparities in utilization of laparoscopic hysterectomies: A nationwide analysis. J. Minim. Invasive Gynecol. 2013, doi:10.1016/j.jmig.2013.08.709.

9. Jacoby, V.L.; Autry, A.; Jacobson, G.; Domush, R.; Nakagawa, S.; Jacoby, A. Nationwide use of laparoscopic hysterectomy compared with abdominal and vaginal approaches. Obstet. Gynecol. 2009, 114, 1041-1048.

10. Stang, A.; Merrill, R.M.; Kuss, O. Nationwide rates of conversion from laparoscopic or vaginal hysterectomy to open abdominal hysterectomy in Germany. Eur. J. Epidemiol. 2011, 26, 125-133.

11. Grantcharov, T.P.; Funch-Jensen, P. Can everyone achieve proficiency with the laparoscopic technique? Learning curve patterns in technical skills acquisition. Am. J. Surg. 2009, 197, 447-449.

12. Coronado, P.J.; Herraiz, M.A.; Magrina, J.F.; Fasero, M.; Vidart, J.A. Comparison of perioperative outcomes and cost of robotic-assisted laparoscopy, laparoscopy and laparotomy for endometrial cancer. Eur. J. Obstet. Gynecol. Reprod. Biol. 2012, 165, 289-294.

13. Weinberg, L.; Rao, S.; Escobar, P.F. Robotic surgery in gynecology: An updated systematic review. Obstet. Gynecol. Int. 2011, 2011, 852061.

14. AAGL Advancing Minimally Invasive Gynecology Worldwide. AAGL position statement: Robotic-assisted laparoscopic surgery in benign gynecology. J. Minim. Invasive Gynecol. 2013, 20,2-9.

15. Mok, Z.W.; Yong, E.L.; Low, J.J.; Ng, J.S. Clinical outcomes in endometrial cancer care when the standard of care shifts from open surgery to robotics. Int. J. Gynecol. Cancer 2012, 22, 819-825.

16. Lau, S.; Vaknin, Z.; Ramana-Kumar, A.V.; Halliday, D.; Franco, E.L.; Gotlieb, W.H. Outcomes and cost comparisons after introducing a robotics program for endometrial cancer surgery. Obstet. Gynecol. 2012, 119, 717-724.

17. Magrina, J.F.; Zanagnolo, V.; Giles, D.; Noble, B.N.; Kho, R.M.; Magtibay, P.M. Robotic surgery for endometrial cancer: Comparison of perioperative outcomes and recurrence with laparoscopy, vaginal/laparoscopy and laparotomy. Eur. J. Gynaecol. Oncol. 2011, 32, 476-480.

(C) 2013 by the authors; licensee MDPI, Basel, Switzerland. This article is an open access article distributed under the terms and conditions of the Creative Commons Attribution license (http://creativecommons.org/licenses/by/3.0/). 